\title{
Fat Embolism Syndrome: Fact or Myth?
}

\author{
Benjamin Stump ${ }^{1} \cdot$ Gerald Weinhouse $^{1}$
}

Published online: 13 April 2016

(C) Springer International Publishing AG 2016

\begin{abstract}
Fat embolism syndrome (FES) is thought to occur most commonly in patients who suffer severe trauma and orthopedic injuries and may be associated with potentially life-threatening pulmonary complications. However, despite its original description hundreds of years ago, it remains a difficult diagnosis to establish and the process by which a fat embolism leads to the clinical syndrome of FES is poorly understood. Some wonder, therefore, if it is a unique syndrome or just part of the spectrum of systemic inflammatory response syndrome (SIRS) common to other critically ill patients. In this review, we will summarize the clinical and experimental evidence that supports FES as a unique clinical condition.
\end{abstract}

Keywords Fat embolism · Fat embolism syndrome · FES . Systemic inflammatory response syndrome $\cdot$ SIRS

\section{Introduction}

Fat emboli (FE) are found most commonly in the setting of severe trauma and orthopedic injuries, with the highest incidence in long bone and pelvic fractures $[1 \bullet \bullet]$. Historical accounts of the first known experimental study about the effects of intravascular FE took place in the seventeenth century, followed by the clinical identification of a fat embolism in a

This article is part of the Topical Collection on Fracture Considerations for the General Surgeon

Gerald Weinhouse

gweinhouse@partners.org

1 Brigham and Women's Hospital, 75 Francis St, Boston, MA 02115, USA human patient 200 years later [2]. More recently, the number of pathologic processes and clinical scenarios believed to be associated with FE have grown substantially. Despite advances in diagnostic techniques and increased understanding of the pathologic processes that lead to fat embolization, the clinical correlate, known as fat embolism syndrome (FES), remains poorly understood.

It is important to recognize the distinction between fat embolism and FES. A fat embolism is the presence of fat within vascular structures. Intravascular fat includes both large fat globules and fat breakdown products such as bioactive free fatty acids. The fat embolism syndrome (FES) constitutes a potentially devastating constellation of clinical signs and symptoms, which are classically characterized as the triad of respiratory insufficiency, neurologic dysfunction, and petechial rash.

Identifying a clear cause-and-effect relationship between fat embolism and the development of the clinical syndrome has been difficult and remains controversial. The diagnosis of FES is imprecise and relies on mostly non-specific clinical findings. The pathway leading from fat embolization to the development of FES is poorly understood and has lead to the question of whether FES is linked directly to circulating fat or represents other sequelae of the original insult.

\section{History}

The first reports of experimental models of fat embolism were documented over 200 years ago [2]. In the latter portion of the nineteenth century, the first known description of fat embolism in humans was reported. In 1862, Zenker identified fat within pulmonary capillaries during post-mortem evaluation of a patient who had sustained a severe crush injury [3]. 
It was not until the 1920s, however, that the first attempts were made to describe the pathophysiology of fat embolism. In 1924, Gauss focused on the mechanism of injury and mechanical disruption of adipose tissue and vascular structures as the cause of intravascular fat. In 1927, Lehman proposed biochemical mechanisms resulting in fat mobilization from body stores and in intravascular fat globule formation, without the requirement of traumatic injury or disruption of tissue or vascular integrity. Both theories, to some extent, will later become the basis for current hypothesized mechanisms leading to fat embolization and FES [2].

Over the latter half of the twentieth century and the beginning of the twenty-first century, increased recognition of FE in the trauma population emerged from armed conflicts including World War II (WWII), the Korean War [4], and the conflict in Vietnam [5, 6]. While the majority of the data produced from theaters of war were merely descriptive of the incidence of FE based upon post-mortem examination, information from Vietnam included reports of arterial hypoxemia that was believed to be most evident in soldiers with femur fractures [6].

\section{Epidemiology}

The incidence of FE varies depending upon the patient population being considered. The most commonly recognized group with FE includes victims of trauma and orthopedic injuries. In 1956, Scully reported an incidence of $93 \%$ during post-mortem evaluation of victims of traumatic injuries from the Korean War [4]. More recently, Mudd et al. reported an incidence of $68 \%$ in patients suffering blunt force trauma [7].

The incidence of FES, while also reported with a wide variability, is substantially lower than the incidence of fat embolism without clinical sequelae. Nineteen percent of major trauma patients admitted to a hospital in Belfast in the 1970s were diagnosed with FES [8•]. An incidence of $29 \%$ was reported by Lindeque et al. in the 1980s in patients with either femur or tibia fractures [9॰]. Bulger et al. reported a retrospective review of records over a 10 -year period from a single level 1 trauma center which suggested a much lower incidence of $0.9 \%$ among patients with long bone fractures $[1 \bullet \bullet]$.

The criteria for establishing the diagnosis of FES differ leading to variability in reporting of its incidence. Additionally, the majority of currently available epidemiologic data are based upon retrospective reviews. Differences in injury severity between study populations also explain the wide variability in reported incidence rates. Mortality rates associated with FES have previously been reported as ranging from 10 to $20 \%$ [10]. More recent data find the mortality to be closer to $7-10 \%$ with the more severe injuries resulting in worse outcomes $[1 \bullet \bullet, 11]$.

In addition to trauma, FES may develop in other clinical contexts such as sickle-cell disease [12-14] and pancreatitis
[15]. A number of cases of FES have also been reported following surgical procedures including liposuction $[16,17]$ and vertebroplasty [18]. Over the last 10 years, cases of respiratory failure due to donor-acquired FES following lung transplant have now been identified as well $[19,20]$.

\section{Pathophysiology}

While the exact mechanisms responsible for FES have yet to be clearly elucidated, two dominant pathophysiologic theories have emerged: (1) mechanical obstruction and (2) biochemical injury.

\section{Mechanical Obstruction Theory}

The mechanical obstruction theory of FES has existed in one form or another for nearly 100 years. This theory is based on the premise that intravascular fat globules associated with platelet activation and fibrin deposition result in pulmonary vascular obstruction similar to other embolic events [21]. This is believed to result in immediate cardiopulmonary decompensation as manifested by hypoxemia within the initial $24 \mathrm{~h}$ of injury. Evidence of acute pulmonary hypertension has been described experimentally [22] and, rarely, clinically [23]. Kao et al. published cardiopulmonary physiology data including levels of vaso-active and inflammatory mediators in eight subjects involved in "crash accidents" who were all found to have pulmonary fat embolism on post-mortem exam [23]. The mean pulmonary artery pressure as measured in the setting of acute pulmonary decompensation ranged from 34 to $48 \mathrm{mmHg}$. Cor pulmonale and acute right heart failure have also been implicated as a possible contributor to mortality due to mechanical obstruction and shock, though specific evidence focusing on right ventricular failure has not been well established.

Proponents of this theory further maintain that intravascular fat globules promote localized endothelial damage resulting in edema and/or hemorrhage. Kim et al. reported the effects of triolein infusion in carotid arteries of cats, either with or without an emulsifying agent [24]. Eightytwo percent of research animals developed evidence of cerebral infarct and hemorrhage when triolein was administered without an emulsifying agent as compared to $9.1 \%$ of animals that received emulsified triolein. The authors postulated that triolein administered without the addition of an emulsifier would either result in macrovascular obstruction by large triolein globules or microscopic capillary obstruction by smaller triolein globules resulting in hemorrhage. There is also experimental evidence that triolein administration results in a pro-inflammatory state [25]. 


\section{Biochemical Injury Theory}

The biochemical injury model has more recently been supported with advances in the understanding of the contribution of vasoactive, thrombotic, and inflammatory mediators to cardiopulmonary and vascular function. Neutral fat is believed to be broken down into free fatty acids resulting in endothelial injury or dysfunction leading to increased vascular permeability and hemorrhage [21].

Experimentally, the infusion of free fatty acids has become widely used in the creation of animal models of fat embolism and FES. A number of reports have been published identifying elevations in multiple inflammatory markers, especially IL-6, as a possible marker for assisting in the diagnosis of FES $[23,26]$.

\section{Diagnosis}

The diagnosis of FES depends on the clinical presentation. It typically occurs within $12-72 \mathrm{~h}$ of the primary insult and is associated with signs and symptoms of multiple organ systems including respiratory compromise, acute changes in neurologic status, hematologic abnormalities (anemia and thrombocytopenia), tachycardia, fever, and, sometimes, a petechial rash [1••]. In 1970, Gurd proposed the use of major and minor criteria in making a clinical diagnosis of FES in the presence of fat macroglobulinemia (Table 1) [8•]. The lists of criteria are non-specific and vary widely in occurrence based upon retrospective data of patients who received a diagnosis of FES $[1 \bullet \bullet]$.

The fat embolism index (FEI) was proposed by Schonfeld et al. in 1983(Table 2) [27•]. Points are awarded to signs of FES with petechial rash assigned the highest number of points for a single finding.

Table 1 Gurd's diagnostic criteria for FES

\begin{tabular}{ll}
\hline Major criteria & Minor criteria \\
\hline $\begin{array}{l}\text { Petechial rash } \\
\begin{array}{c}\text { Respiratory symptoms plus } \\
\text { bilateral signs with positive } \\
\text { radiographic findings }\end{array}\end{array}$ & Tachycardia \\
$\begin{array}{c}\text { Cerebral signs unrelated } \\
\text { to head injury or any } \\
\text { other condition }\end{array}$ & Retinal changes (fat or petechiae) \\
& Urinary changes (anuria, oliguria, \\
fat globules) \\
Sudden drop in hemoglobin level \\
Sudden thrombocytopenia \\
High erythrocyte sedimentation rate \\
Fat globules in sputum
\end{tabular}

Diagnosis requires one major and four minor criteria in the presence of fat macroglobulinemia $[8 \bullet]$
Table 2 Schonfeld's fat embolism index (FEI) score

Fat embolism index score

\begin{tabular}{ll}
\hline Petechial rash & 5 \\
Diffuse alveolar infiltrates & 4 \\
Hypoxemia (arterial $\left.\mathrm{PO}_{2}<70 \mathrm{mmHg}\right)$ & 3 \\
Confusion & 1 \\
Fever $\geq 38^{\circ} \mathrm{C}\left(100.4{ }^{\circ} \mathrm{F}\right)$ & 1 \\
Heart rate $\geq 120 / \mathrm{min}$ & 1 \\
Respiratory rate $\geq 30 / \mathrm{min}$ & 1 \\
\hline
\end{tabular}

Diagnosis requires an FEI score of 5 points or more accumulated within 3 days of hospitalization [27•]

Lindeque et al. suggested far more lenient criteria based on pulmonary signs and symptoms. [9•] According to the investigators, a diagnosis of FES should be made if the patient has a $\mathrm{PO}_{2}$ of less than $60 \mathrm{mmHg}$; $\mathrm{PCO}_{2}$ greater than 55 or a $\mathrm{pH}$ less than 7.3; a sustained respiratory rate $>35$ breaths per minute despite adequate sedation; or increased work of breathing as identified by dyspnea, use of accessory respiratory muscles, and tachycardia in combination with anxiety. All three proposed sets of criteria vary with regard to their sensitivity and specificity. There has not been a documented effort to compare the performance of one set of diagnostic criteria over another.

A variety of diagnostic techniques have been used in an attempt to support the clinical findings with additional objective data to improve the accuracy of making a diagnosis of FES. Chest radiology was not found to be either sensitive or specific for FES as a wide variety of findings including ground glass, septal thickening, nodularity, and consolidation as presented in one retrospective review [28]. These authors reported that the use of contrast enhancement rarely yielded evidence of filling defects supportive of vascular obstruction.

Neuroradiology has had more success in establishing the diagnosis of FES. While CT is less helpful, magnetic resonance imaging (MRI), particularly diffusion-weighted protocols, can identify the "starfield" pattern that is representative of scattered cytotoxic edema [29-31]. The use of diffusionweighted images has also been proposed to provide prognostic information as well. Decaminada et al. reported that a patient with increased evidence of cytotoxic edema and diffusion restriction had a worse outcome when compared to a patient without diffusion restriction, though this is based on case reports only [32]. The starfield pattern likely represents an early finding in the development of cerebral FES with additional non-specific abnormalities including confluent areas of cytotoxic edema and white matter petechial hemorrhages reported as well [33•].

Ultrasound has been used as a method of identifying fat embolization. Trans-esophageal echocardiogram (TEE) has been used intraoperatively in order to monitor for FE as 
represented by intracardiac echogenic material that is observed during orthopedic procedures [34-36]. There is limited data correlating TEE with the risk of developing FES. The results of a small prospective study involving 24 patients proposed that larger emboli as identified on TEE $(>10 \mathrm{~mm})$ correlated with an increased incidence of FES and death [34]. That same study also observed that earlier surgical intervention may have yielded smaller emboli. Despite the lack of evidence for routine use in monitoring or diagnosing fat emboli, TEE has gained popularity in research as a method of identifying surgical procedures for orthopedic repair that result in less embolization [35].

In addition to TEE, transcranial Doppler (TCD) has been used as a non-invasive method to identify fat emboli. Investigations using contrast-enhanced TCD have reported findings comparable in sensitivity and specificity to TEE in the diagnosis of right-to-left intracardiac shunt (RLS), such as in the case of a patent foramen ovale (PFO) [37]. TCD has now been used to identify emboli traveling to the CNS [38]. Similar to the identification of embolization on TEE, the use of TCD to risk stratify patients for the development of FES is limited; however, none of the patients with a negative exam for RLS developed neurologic abnormalities in one recent prospective study. Conversely, images obtained from patients with RLS yielded evidence of embolization with the subsequent increased rate of neurologic abnormalities within this patient population.

Biomarkers have been introduced as a possible means of predicting the development of FES. Research aimed at evaluating various vasoactive and inflammatory mediators in the setting of FES has found significant increases in phospholipase $A_{2}\left(\mathrm{PLA}_{2}\right)$, nitric oxide (NO), methylguanine (MG), TNF-A, IL-10, IL-1B, and IL-6 [23]. While none of these mediators are specific to FES, IL-6 has been proposed for use in identifying early FES [26]. No specific biomarker has, as of yet, been validated as predicting the risk of developing FES or in clearly assisting with the diagnosis of FES.

Information gained from broncho-alveolar lavage (BAL) may contribute to the diagnosis of FES. Roger et al. reported BAL data obtained from 32 trauma patients who met variable levels of criteria for FES [39]. Oil Red O stain was used to identify fat within pulmonary macrophages. The seven patients who met the full criteria for FES had significantly more fat-laden macrophages as compared to other trauma patients who did not meet clinical criteria for FES, non-trauma patients with pulmonary infiltrates, and normal controls without a history of trauma and without known pulmonary pathology. Similarly, elevated numbers of fat-laden macrophages have also been identified in sickle-cell patients with acute chest syndrome as compared to sickle-cell patients without acute chest syndrome as well as normal controls [40]. The use of BAL has also been reported to identify significantly higher levels of intraalveolar cholesterol in patients with FES in comparison to other patients with acute lung injury and normal controls [41]. In the orthopedic surgery population, the use of BAL in diagnosing FES is less clear as there was no significant difference in the percentage of lipid-laden macrophages in patients with recent intramedullary reaming without clinical criteria for FES as compared to those who met criteria for the diagnosis of FES [42].

A number of additional methods of identifying fat embolization have been proposed as possible tools for assisting with the diagnosis of FES. The finding of fat globules in blood sampled via pulmonary artery catheter from the pulmonary circulation has been documented in several investigations [43-45]. However, similar to the identification of embolic events based upon ultrasound, the correlation between blood sample findings and clinical manifestations of FES is not well supported. The identification of lipuria (which has been labeled as a common finding in FES) is even less well supported as a method of detecting FE or correlating with FES.

\section{Treatment}

Supportive care remains the treatment of FES. As hypoxemia is the most common finding in FES, early recognition and initiation of cardiopulmonary support not specific to the underlying pathology are most important $[1 \bullet \cdot, 46]$. There remains a lack of evidence for additional interventions or medications that promote FES resolution.

Early fixation of bone fractures and the timing of surgical repair after the initial injury as well as specific surgical repair techniques may reduce the risk of FES. The incidence of pulmonary complications are increased with delayed fixation $[47,48,49 \bullet$. Bone et al. reported an increase in FES associated with delayed fixation of femur fractures beyond $24 \mathrm{~h}$ in patients with multiple fractures [49•]. The diagnosis of FES was based upon changes in mental status, arterial hypoxemia, and interstitial infiltrates on chest radiographs. As with many other reports, arterial hypoxemia was the most common pulmonary complication across all cohorts.

More recently, a retrospective review of 750 patients with femur fractures identified significantly fewer pulmonary complications (including pneumonia, PE, or acute respiratory distress syndrome (ARDS)) in patients with definitive surgical correction within $24 \mathrm{~h}$ of the initial injury [50•]. The authors stratified their data to account for overall injury severity, abdominal injuries, and chest injuries. While the presence of chest injuries increased the risk of pulmonary complications overall when compared to patients without chest injuries, there was a clear benefit of definitive femur fracture stabilization within $24 \mathrm{~h}$. The majority of the pulmonary complications reported were in the form of pneumonia, while only a small portion was made up of patients with ARDS. 
In addition to the timing of surgical correction, the type of orthopedic procedure, particularly in long bone fractures, may affect the incidence of FE and FES. High intramedullary pressure during surgical repair of femoral fractures involving intramedullary reaming is theorized to result in intravasation of marrow material precipitating embolic events and increasing the risk of FES. Continuous intramedullary irrigation and aspiration may improve intraprocedural pressure parameters and are believed to limit marrow intravasation into systemic circulation in animal studies [51]. Intraprocedural echocardiogram images in a human study evaluating the extent to which the right atrium filled with echogenic material suggested that reamer-irrigator-aspirator (RIA) use led to less embolization [52]. Hartsock et al. randomized 19 patients to RIA vs. standard reaming. The authors reported levels of various cytokines (serum IL-2, IL-6, IL-8, IL-10, and TNF; BAL IL-1B and IL-8) [54]. There was a statistically significant increase in post-procedural IL-10 and a similar trend in IL6 in the standard reaming group. However, the use of RIA appears to have had little effect on clinical outcomes. Retrospective review of 156 patients between 2005 and 2006 who underwent conventional reaming vs RIA did not identify significant differences in length of hospital stay, length of ICU stay, or duration of mechanical ventilation [53].

Corticosteroids have been proposed as an intervention to limit the progression of inflammation believed to result in organ dysfunction in FES. Over the last 50 years, several small studies have reported evidence that prophylactic corticosteroids limit the degree of hypoxemia in orthopedic injury patients $[27 \bullet, 54,55]$. In 2009, Bederman et al. published a metaanalysis evaluating the use of corticosteroids [56•]. The included studies identified a decrease in incidence of FES and hypoxemia but not in mortality in patients with long bone fractures. The authors recognized the difficulty in conveying clear recommendations based upon the meta-analysis results, due to the variability in criteria used to diagnose FES as well as the variability in steroid dosing. Regardless, some believe the risk of systemic corticosteroids to be low enough to recommend the use of corticosteroids in high-risk orthopedic injuries.

\section{The Controversy}

Despite having been described centuries ago, FES remains a controversial diagnosis. The question at the center of the debate is whether FES exists as a distinct entity or is the constellation of signs and symptoms common to many clinical situations including trauma.

FES is classically defined by the triad of respiratory insufficiency, neurologic dysfunction, and petechial rash. However, it is uncommon to find all three features of the triad in patients diagnosed with FES. Bulger et al. identified hypoxemia as the dominant finding in $96 \%$ of the cases that they reviewed over a 10 -year period $[1 \bullet \bullet]$. Mental status changes were documented in $59 \%$ of cases while petechial rash was only noted in $33 \%$ of cases. The variability in presentation particularly in the setting of trauma and orthopedic injuries resulted in the development of diagnostic criteria by Gurd in the 1970s (Table 1) [8•] and, subsequently, Schonfeld in the 1980s (Table 2) [27•]. Lindeque advocated for excluding all findings other than those related to respiratory insufficiency out of concern for excluding a potentially devastating diagnosis [9•]. This more liberal definition of FES may account for some clinicians' skepticism of FES as a distinct entity as there will clearly be patients diagnosed with FES who may have another cause of respiratory failure.

The pathophysiology contributing to FES remains elusive. Initially believed to be the direct consequence of circulating fat globules, it is now recognized that FE occur in a variety of clinical settings with fewer patients developing FES. This raises the question of whether or not FES is a result of FE or, rather, represents sequelae of the initial insult without the influence of circulating fat globules. Experimental models and clinical scenarios implicate circulating fat, particularly in the form of bioactive products of fat metabolism, as contributing to inflammation and predisposing to organ dysfunction.

Finally, while based largely on case reports, MR imaging has emerged as a tool for the diagnosis of cerebral FES. The starfield pattern is the most well-documented radiographic finding in patients with neurologic dysfunction and medical conditions or injuries known to predispose to FES. While not specific to FES, the starfield pattern is objective evidence that may support the involvement of FE in end-organ dysfunction particularly in the setting of trauma and orthopedic injuries. Other diagnostic modalities have been used to evaluate for FE including ultrasound and blood sampling; however, they do not consistently correlate with the clinical sequelae of FES.

\section{Conclusion}

FE are found predominantly, but not exclusively, in victims of trauma and orthopedic injuries and surgeries. The clinical syndrome, FES, observed in some of these patients remains a poorly understood but potentially devastating complication of multiple injuries and some disease processes. Diagnosing FES relies predominantly on non-specific clinical findings such as respiratory and neurologic dysfunction, which are common in critical illness. However, the consistency of these findings and prevalence in a specific clinical context, the findings from animal models of circulating fat or free fatty acid, some more specific clinical findings such as a petechial rash and the presence of the starfield pattern on MRI of the brain, 
and several centuries of recorded clinical observations support FES as a true unique clinical syndrome.

\section{Compliance with Ethical Standards}

Conflict of Interest Drs. Stump and Weinhouse declare no conflicts of interest.

Human and Animal Rights and Informed Consent This article does not contain any studies with human or animal subjects performed by any of the authors.

\section{References}

Papers of particular interest have been highlighted as:

- Of importance

•- Of major importance

1.• Bulger EM, Smith DG, Maier RV, Jurkovich GJ. Fat embolism syndrome. A 10-year review. Arch Surg. 1997;132:435-9. 10 year retrospective review of 3026 patient records from a single trauma center including discussion of injury characteristics, methods of diagnosis, and treatments in patients with FES.

2. Talbot M, Schemitsch EH. Fat embolism syndrome: history, definition, epidemiology. Injury. 2006;37 Suppl 4:S3-7.

3. Zenker F. Beitrage zur normalen und pathologischen anatomic der lunge. J Braunsdorf. 1862;31.

4. Scully RE. Fat embolism in Korean battle casualties; its incidence, clinical significance, and pathologic aspects. Am J Pathol. 1956;32: 379-403.

5. Cloutier CT, Lowery BD, Strickland TG, Carey LC. Fat embolism in Vietnam battle casualties in hemorrhagic shock. Mil Med. 1970;135:369-73.

6. Collins JA, Gordon Jr WC, Hudson TL, Irvin Jr RW, Kelly T, Hardaway 3rd RM. Inapparent hypoxemia in casualties with wounded limbs: pulmonary fat embolism? Ann Surg. 1968;167: 511-20.

7. Mudd KL, Hunt A, Matherly RC, et al. Analysis of pulmonary fat embolism in blunt force fatalities. J Trauma. 2000;48:711-5.

8. Gurd AR. Fat embolism: an aid to diagnosis. J bone Joint Surg Br. 1970;52:732-7. Original diagnostic criteria for FES.

9. Lindeque BG, Schoeman HS, Dommisse GF, Boeyens MC, Vlok AL. Fat embolism and the fat embolism syndrome. A double-blind therapeutic study. J Bone Joint Surg Br. 1987;69:128-31. Proposed set of diagnostic criteria with increased sensitivity in the identification of FES.

10. Peltier LF, Collins JA, Evarts CM, Sevitt S. A panel by correspondence. Fat embolism. Arch Surg. 1974;109:12-6.

11. Fabian TC, Hoots AV, Stanford DS, Patterson CR, Mangiante EC. Fat embolism syndrome: prospective evaluation in 92 fracture patients. Crit Care Med. 1990;18:42-6.

12. Dhakal LP, Bourgeois K, Barrett KM, Freeman WD. The "starfield" pattern of cerebral fat embolism from bone marrow necrosis in sickle cell crisis. Neurohospitalist. 2015;5:74-6.

13. Graff DM, Owen E, Bendon R, Bertolone S, Raj A. Distinctive acellular lipid emboli in hemoglobin SC disease following bone marrow infarction with parvovirus infection. Case Rep Hematol. 2015;2015:328065.
14. Graham JK, Mosunjac M, Hanzlick RL, Mosunjac M. Sickle cell lung disease and sudden death: a retrospective/prospective study of 21 autopsy cases and literature review. Am J Forensic Med Pathol. 2007;28:168-72.

15. Guardia SN, Bilbao JM, Murray D, Warren RE, Sweet J. Fat embolism in acute pancreatitis. Arch Pathol Lab Med. 1989;113:503-6.

16. Byeon SW, Ban TH, Rhee CK. A case of acute fulminant fat embolism syndrome after liposuction surgery. Tuberc Respir Dis. 2015;78:423-7.

17. Wang HD, Zheng JH, Deng CL, Liu QY, Yang SL. Fat embolism syndromes following liposuction. Aesthet Plast Surg. 2008;32: 731-6.

18. Ahmadzai H, Campbell S, Archis C, Clark WA. Fat embolism syndrome following percutaneous vertebroplasty: a case report. Spine J Off J N Am Spine Soc. 2014;14:e1-5.

19. Jacob S, Courtwright A, El-Chemaly S, et al. Donor-acquired fat embolism syndrome after lung transplantation. Eur J Cardiothor Surg Off J Eur Assoc Cardiothorac Surg 2015

20. Padilla J, Jorda C, Penalver JC, Ceron J, Escriva J, Vera-Sempere F. Donor fat embolism and primary graft dysfunction after lung transplantation. Ann Thorac Surg. 2007;84:e4-5.

21. Husebye EE, Lyberg T, Roise O. Bone marrow fat in the circulation: clinical entities and pathophysiological mechanisms. Injury. 2006;37 Suppl 4:S8-18.

22. Winn R, Maunder R, Harlan J. Lung lymph flow after bone marrow injection into goats was reduced by indomethacin. J Appl Physiol. 1987;62:762-7.

23. Kao SJ, Yeh DY, Chen HI. Clinical and pathological features of fat embolism with acute respiratory distress syndrome. Clin Sci. 2007;113:279-85.

24. Kim YW, Kim HJ, Choi SH, Cho B, Hwangbo L, Kim DC. Hemorrhage in cerebral fat embolisms in a cat model using triolein dependent on the physical properties of triolein. Jpn J Radiol. 2014;32:30-7.

25. McIff TE, Poisner AM, Herndon B, et al. Fat embolism: evolution of histopathological changes in the rat lung. J Orthop Res Off Publ Orthop Res Soc. 2010;28:191-7.

26. Prakash S, Sen RK, Tripathy SK, Sen IM, Sharma RR, Sharma S. Role of interleukin- 6 as an early marker of fat embolism syndrome: a clinical study. Clin Orthop Relat Res. 2013;471:2340-6.

27. Schonfeld SA, Ploysongsang Y, DiLisio R, et al. Fat embolism prophylaxis with corticosteroids. A prospective study in high-risk patients. Ann Intern Med. 1983;99:438-43. Prospective randomized control trial investigating the use of prophylactic corticosteroids in high risk patients with long bone fractures and the presentation of Fat Embolism Index Score as a diagnostic tool.

28. Malagari K, Economopoulos N, Stoupis C, et al. High-resolution CT findings in mild pulmonary fat embolism. Chest. 2003;123: 1196-201.

29. Gregorakos L, Sakayianni K, Hroni D, et al. Prolonged coma due to cerebral fat embolism: report of two cases. J Accid Emerg Med. 2000;17:144-6.

30. Takahashi M, Suzuki R, Osakabe Y, et al. Magnetic resonance imaging findings in cerebral fat embolism: correlation with clinical manifestations. J Trauma. 1999;46:324-7.

31. Kellogg RG, Fontes RB, Lopes DK. Massive cerebral involvement in fat embolism syndrome and intracranial pressure management. $\mathrm{J}$ Neurosurg. 2013;119:1263-70.

32. Decaminada N, Thaler M, Holler R, Salsa A, Ladiges C, Rammlmair G. Brain fat embolism. A report of two cases and a brief review of neuroimaging findings. Neuroradiol J. 2012;25: 193-9.

33. Kuo KH, Pan YJ, Lai YJ, Cheung WK, Chang FC, Jarosz J. Dynamic MR imaging patterns of cerebral fat embolism: a systematic review with illustrative cases. AJNR Am J Neuroradiol. 
2014;35:1052-7. Review of MR imaging patterns in cerebral fat embolization.

34. Pell AC, Christie J, Keating JF, Sutherland GR. The detection of fat embolism by transoesophageal echocardiography during reamed intramedullary nailing. A study of 24 patients with femoral and tibial fractures. J Bone Joint Surg Br. 1993;75:921-5.

35. Zhao J, Zhang J, Ji X, Li X, Qian Q, Xu Q. Does intramedullary canal irrigation reduce fat emboli? A randomized clinical trial with transesophageal echocardiography. J Arthroplast. 2015;30:451-5.

36. Koessler MJ, Pitto RP. Fat and bone marrow embolism in total hip arthroplasty. Acta Orthop Belg. 2001;67:97-109.

37. Klotzsch C, Janssen G, Berlit P. Transesophageal echocardiography and contrast-TCD in the detection of a patent foramen ovale: experiences with 111 patients. Neurology. 1994;44:1603-6.

38. Forteza AM, Koch S, Campo-Bustillo I, et al. Transcranial Doppler detection of cerebral fat emboli and relation to paradoxical embolism: a pilot study. Circulation. 2011;123:1947-52.

39. Roger N, Xaubet A, Agusti C, et al. Role of bronchoalveolar lavage in the diagnosis of fat embolism syndrome. Eur Respir J. 1995;8: 1275-80.

40. Godeau B, Schaeffer A, Bachir D, et al. Bronchoalveolar lavage in adult sickle cell patients with acute chest syndrome: value for diagnostic assessment of fat embolism. Am J Respir Crit Care Med. 1996;153:1691-6.

41. Karagiorga G, Nakos G, Galiatsou E, Lekka ME. Biochemical parameters of bronchoalveolar lavage fluid in fat embolism. Intensive Care Med. 2006;32:116-23.

42. Aoki N, Soma K, Shindo M, Kurosawa T, Ohwada T. Evaluation of potential fat emboli during placement of intramedullary nails after orthopedic fractures. Chest. 1998;113:178-81.

43. Masson RG, Ruggieri J. Pulmonary microvascular cytology. A new diagnostic application of the pulmonary artery catheter. Chest. 1985;88:908-14.

44. Adolph MD, Fabian HF, el-Khairi SM, Thornton JC, Oliver AM. The pulmonary artery catheter: a diagnostic adjunct for fat embolism syndrome. J Orthop Trauma. 1994;8:173-6.

45. Gitin TA, Seidel T, Cera PJ, Glidewell OJ, Smith JL. Pulmonary microvascular fat: the significance? Crit Care Med. 1993;21:673-7.
46. Georgopoulos D, Bouros D. Fat embolism syndrome: clinical examination is still the preferable diagnostic method. Chest. 2003;123:982-3.

47. Beckman SB, Scholten DJ, Bonnell BW, Bukrey CD. Long bone fractures in the polytrauma patient. The role of early operative fixation. Am Surg. 1989;55:356-8.

48. Behrman SW, Fabian TC, Kudsk KA, Taylor JC. Improved outcome with femur fractures: early vs. delayed fixation. J Trauma. 1990;30:792-7. discussion 7-8.

49. Bone LB, Johnson KD, Weigelt J, Scheinberg R. Early versus delayed stabilization of femoral fractures. A prospective randomized study. J Bone Joint Surg Am. 1989;71:336-40. Prospective randomized trial evaluating early vs. late femur fracture stabilization.

50. Nahm NJ, Como JJ, Wilber JH, Vallier HA. Early appropriate care: definitive stabilization of femoral fractures within 24 hours of injury is safe in most patients with multiple injuries. J Trauma. 2011;71: 175-85. Retrospective review of 750 patients with evidence supporting early femur fracture stabilization.

51. Danckwardt-Lilliestrom G, Lorenzi GL, Olerud S. Intramedullary nailing after reaming. An investigation on the healing process in osteotomized rabbit tibias. Acta Orthop Scand Suppl. 1970;134:1-78.

52. Volgas DA, Burch T, Stannard JP, Ellis T, Bilotta J, Alonso JE. Fat embolus in femur fractures: a comparison of two reaming systems. Injury. 2010;41 Suppl 2:S90-3.

53. Streubel PN, Desai P, Suk M. Comparison of RIA and conventional reamed nailing for treatment of femur shaft fractures. Injury. 2010;41 Suppl 2:S51-6.

54. Alho A, Saikku K, Eerola P, Koskinen M, Hamalainen M. Corticosteroids in patients with a high risk of fat embolism syndrome. Surg, Gynecol Obstet. 1978;147:358-62.

55. Babalis GA, Yiannakopoulos CK, Karliaftis K, Antonogiannakis E. Prevention of posttraumatic hypoxaemia in isolated lower limb long bone fractures with a minimal prophylactic dose of corticosteroids. Injury. 2004;35:309-17.

56. Bederman SS, Bhandari M, McKee MD, Schemitsch EH. Do corticosteroids reduce the risk of fat embolism syndrome in patients with long-bone fractures? A meta-analysis. Can J Surg J Can Chir. 2009;52:386-93. Meta-analysis of available data regarding the use of prophylactic corticosteroids. 